\title{
Use of 3D Printed PLA for Diffuse Optics
}

\author{
R. Re ${ }^{1,2}$, I. Pirovano ${ }^{1}$, C. Amendola ${ }^{1}$, M. Lacerenza ${ }^{1}$, D. Contini ${ }^{1}$, L. Spinelli ${ }^{2}$, R. Cubeddu ${ }^{1}$ and A. Torricelli ${ }^{1,2}$ \\ ${ }^{I}$ Dipartimento di Fisica, Politecnico di Milano, Piazza Leonardo da Vinci 32, 20133 Milan, Italy; ${ }^{2}$ Istituto di Fotonica e Nanotecnologie, \\ Consiglio Nazionale delle Ricerche, Piazza Leonardo da Vinci 32, 20133 Milan, Italy \\ rebecca.re@polimi.it
}

\begin{abstract}
D filament printers are nowadays used in diffuse optics for custom probes or phantoms boxes fabrication. We observed that not all the PLA filaments are suitable for NIRS and DCS applications. (C) 2020 The Author(s)
\end{abstract}

\subsection{D printing in diffuse optics with PLA: the problem}

In the wide additive manufacturing world, the fused filament 3D printers (FDM), are those who are more spread because of the low printer and filament costs and the usage simplicity compared to other 3D printer techniques such as stereolithography (SLA) or selective laser sintering (SLS). One of the most used filaments is made of poly lactic acid (PLA), a thermoplastic aliphatic polyester derived from corn starch, tapioca roots, or sugarcane. This material was already widely characterized from a mechanical and thermal point of view but there is a lack of knowledge regarding its optical properties. We have just a study on the material index of refraction [1] and on the IR transmittance spectrum when different PLA blending are created [2]; whereas no information about the capacity of a printed PLA piece to transmit laser light at different wavelengths is present. This aspect is crucial in diffuse optics (DO), where a knowledge of the spectrum is required when probes or holders for optical fibers, phantom boxes or components for detector's optics are 3D printed with PLA. This is in particular true when single photon counting techniques are used in the detection chain, such as in time-domain near infrared spectroscopy (TD-NIRS) and diffuse correlation spectroscopy (DCS). The aim of this preliminary work is to understand if PLA black filaments for FDM can be simply applied in DO or if they need a priori characterization.

\section{PLA optical spectrum}

Samples of two different commercial black PLA filaments: 3D iTALY (3Di, 3D iTALY, Roma, Italy) and FILOALFA (FA, FILOALFA, Ozzero, Italy), were printed with a FDM printer (Sharebot NG, Nibionno, Italy; Filament diameter $1.75 \mathrm{~mm})$. The chosen geometry was a parallelepiped $\left(40 \times 40 \times 10 \mathrm{~mm}^{3}\right)$, with a $20 \%$ rectilinear infill and 4 top and bottom layers. Two different samples were printed for each material: one with the layers grown perpendicular $(\perp)$ to the laser beam and the other with parallel (II) layers to the laser beam, Fig. 1(a). The samples were tested with a TD-NIRS device [3] which employs supercontinuum laser sources and SIPM detectors providing a 14 wavelengths spectrum. The measurements were performed in a transmittance geometry across the $10 \mathrm{~mm}$ side. We perform 10 repeated measurements per each wavelength, with $1 \mathrm{~s}$ acquisition time and a goal of $4 \cdot 10^{5}$ counts/s. As we can see in Fig. 1(b), the 3Di sample with " $\perp$ " configuration is the only one that doesn't allow the light to pass through at all the wavelengths.
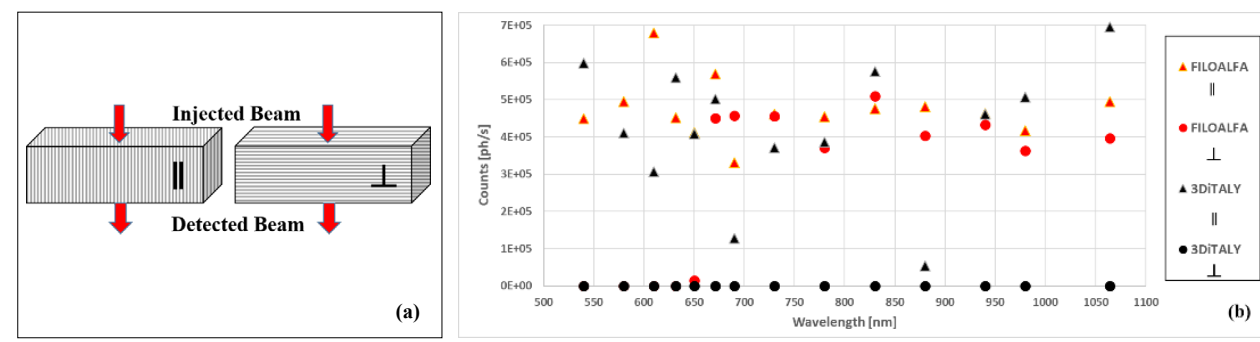

Fig. 1. (a) Structure of the 3D printed PLA samples; (b) transmittance results for the 4 samples.

\section{A practical example for TD-NIRS}

We tested the previous findings directly on a printed custom probe for a TD-NIRS system. The instrument is described in the paper from Re et al. [4], but improved with high power pulsed diode laser heads and cooled hybrid photomultipliers detectors (PicoQuant, Berlin, Germany) for a resulting responsivity of $2.97 \cdot 10^{-8} \mathrm{~m}^{2} \cdot \mathrm{sr}$ at $689 \mathrm{~nm}$ (RED) and $2.07 \cdot 10^{-8} \mathrm{~m}^{2} \cdot \mathrm{sr}$ at $828 \mathrm{~nm}$ (IR). We printed the holder for the $90^{\circ}$ bended optical fibers described in [4] 
with just one interfiber distance for simplicity $(\rho=30 \mathrm{~mm})$ with both the PLA materials previously cited ( $20 \%$ rectilinear infill, 2 top and bottom layers). We tested in term of counts/s the detected signal in two cases: the probe placed 1) over a metallic surface and 2) over a totally black absorbing Polyvinyl chloride (PVC) surface (Fig. 2(a), inset). The laser beam is going to encounter both the $\perp$ and $\|$ structures, so the expected effect should be a mix of the two. In both the cases we perform 30 repeated measurements of $1 \mathrm{~s}$ acquisition rate, for both the wavelengths with $3 \mathrm{~mW}(8.5 \mathrm{~mW})$ of power for the RED (IR). From the results in Fig. 2(a), it can be observed that we have signal just for the FA filament. The counts are higher in the case of metallic surface because of likely reflections transmitted inside the material. We did also a measurement on a calibrated phantom in order to understand the effect of this unwanted signal through the probe on the retrieval of the optical properties. The injected power was set in order to have $10^{6}$ counts/s for each acquisition. We performed 30 repeated measurements for each probe. The values found for the absorption and scattering coefficients were calculated as the average over the 30 repetitions. The standard deviations were negligible. The differences between coefficients found for the two materials reached also the $10 \%$, consequence of the bad quality of the fit procedure with the solution of the diffusion equation for a semiinfinite homogeneous medium. The impact of having a "transparent" PLA is then not negligible.

\section{A practical example for DCS}

We perform the same measurements described in the paragraph before on the metallic and the PVC surfaces (Fig. 2(b), inset), using a home-built DCS system. It employs a continuous wave laser @ 784 nm with $120 \mathrm{~mW}$ of maximum power, and coherence length higher than $8 \mathrm{~m}$ (Toptica photonics, Germany). For the detection chain, it employs a single photon avalanche diode detector (Excelitas thecnology, Canada) and a digital correlator (ALV, Germany). Also in this case, as shown in Fig. 2(b), we can notice a significant signal amount only for the FA probe (circles). Furthermore, we found a linear behavior of the photons count in function of the injected power $\left(\mathrm{R}^{2}=0.99\right.$ of the linear fit). We then tested both the probes in a real measurement on a liquid phantom $\left(\mu_{\mathrm{a}}=0.11 \mathrm{~cm}^{-1}, \mu_{\mathrm{s}}=10.6\right.$ $\mathrm{cm}^{-1}$, diffusion coefficient $\left.\mathrm{D}_{\mathrm{b}}=10^{-8} \mathrm{~cm}^{-2} / \mathrm{s} @ 785 \mathrm{~nm}\right)$. We observed that from the autocorrelation functions we had two different $\mathrm{D}_{\mathrm{b}}$. The one for the 3Di probe (more similar to the nominal one) was two times the one measured with FA probe. This non negligible error is due to the signal artifact created by the unwanted crosstalk inside the material.
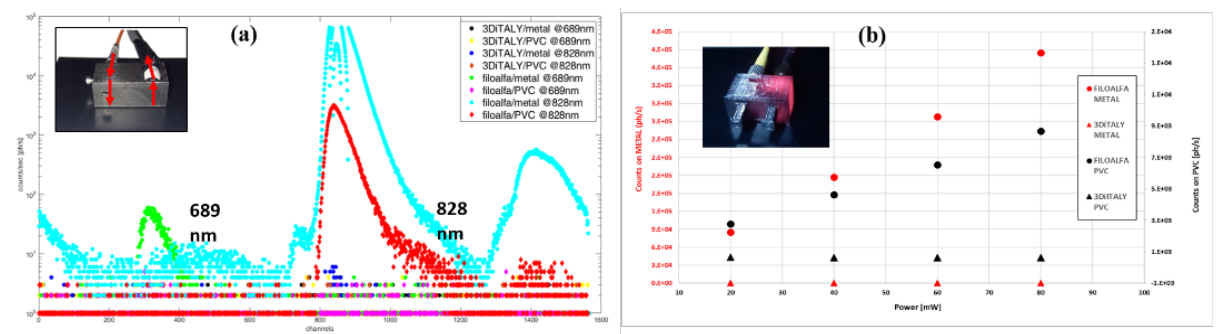

Fig. 2. (a) TD-fNIRS reflectance curves for the different probes and surfaces. (b) DCS photon counts detected with different probes and surfaces in function of the injected power.

\section{Conclusion}

From our preliminary results, the PLA materials need a preliminary optical characterization before being applied in DO applications, such as NIRS or DCS, in order to avoid the presence of unwanted signal through the material itself. Different manufactures seem to have filaments with different optical behavior in term of photons transmission at different wavelengths. At this moment, the best practice it is to perform an optical characterization (transmittance signal at the desired wavelength) of the printed material in the geometry of the desired final object. We are working in the direction of creating a standard for what concern different percentage of infill, direction and type of the infill and kind/color of the material employed.

\section{References}

[1] M. H. Hutchinson et al. "Optical properties of polylactides," J. Polym. Environ. 14, 119-124 (2006).

[2] C. L. Jun, "Reactive blending of biodegradable polymers: PLA and starch," J. Polym. Environ. 8, 33-37 (2000).

[3] E. Zerbini et al., "Optical properties, ethylene production and softening in mango fruit," Postharvest Biol. Technol., 101, 58-65 (2015).

[4] R. Re et al. "Time Domain Near Infrared Spectroscopy Device for Monitoring Muscle Oxidative Metabolism: Custom Probe and In Vivo Applications," Sensors 18, 1-13 (2018). 